\title{
ELUCIDAÇÃO DA QUIRALIDADE INDUZIDA NA MOLÉCULA DANSILGLICINA NA COMPLEXAÇÃO COM A PROTEÍNA ALBUMINA DO SORO HUMANO (HSA)
}

\author{
Aguinaldo Robinson de Souza ${ }^{\mathrm{a}}$, Izabelle Amorim Ferreira Boza ${ }^{\mathrm{a}, *, \odot}$, Valdecir Farias Ximenes $^{\mathrm{a}}$, Maurício Ikeda Yoguin ${ }^{\mathrm{a}}$, \\ María-José Dávila-Rodriguez ${ }^{\mathrm{c}, \mathrm{d}}$, Nelson Henrique Morgon ${ }^{\mathrm{b}}$ e Ignez Caracelli ${ }^{\mathrm{c}}$ \\ aDepartamento de Química, Faculdade de Ciências, Universidade Estadual Paulista, 17033-360 Bauru - SP, Brasil \\ bepartamento de Fisico-Química, Universidade Estadual de Campinas, 13083-970 Campinas - SP, Brasil \\ 'Departamento de Física, Universidade Federal de São Carlos, 13565-905 São Carlos - SP, Brasil \\ ${ }^{\text {d} D e p a r t a m e n t o ~ d e ~ Q u i ́ m i c a, ~ U n i v e r s i d a d e ~ F e d e r a l ~ d e ~ S a ̃ o ~ C a r l o s, ~ 13565-905 ~ S a ̃ o ~ C a r l o s ~-~ S P, ~ B r a s i l ~}$
}

Recebido em 29/06/2018; aceito em 06/12/2018; publicado na web em 16/01/2019

\begin{abstract}
ELUCIDATION OF THE INDUCED CHIRALITY OF DANSYLGLYCINE BY ITS INTERACTION WITH HUMAN SERUM ALBUMIN. Human serum albumin (HSA) plays an important role in the transport of a wide variety of substances, including compounds with pharmacological properties. The dansylglycine $(\mathrm{DanG})$ is a fluorescent amino acid derivative specific for the site II of HSA. This work aimed to elucidate the induction of chirality in the DanG due to its bonding to the HSA. Theoretical electronic circular dichroism spectra (ECDs) were simulated using the Density Functional Theory (DFT) and the implicit Solvation Model based on Density (SMD). The DanG-HSA complexation resulted in the appearance of a positive ECD spectrum centered at $346 \mathrm{~nm}$. Focusing on the dihedral angles between the $-\mathrm{N}\left(\mathrm{CH}_{3}\right)_{2}$ group bounded to the naphthalene ring, the potential energy surface (PES) of the DanG was obtained. Analysis of the various conformations obtained revealed that the calculated dihedral angle $\left(150^{\circ}\right)$ is in agreed with the experimental ECD spectrum. In addition, we observed that the nitrogen atom of the $-\mathrm{N}\left(\mathrm{CH}_{3}\right)_{2}$ group presented the greatest contribution to the HOMO-LUMO transition that gives rise to the $\mathrm{n} \rightarrow \pi^{*}$ electronic transition involved in the generation of the ECD signal. Molecular docking analysis of the complexation between DanG and HSA revealed a conformation with a dihedral angle similar to that obtained by DFT.
\end{abstract}

Keywords: human serum albumin; DFT; electronic circular dichroism; dansylglycine.

\section{INTRODUÇÃO}

A Albumina do Soro Humano (HSA) confere um importante papel no transporte de substâncias com propriedades farmacológicas devido à sua alta concentração no plasma e especificidade, colocando-a como a proteína fundamental responsável na implicação farmacocinética dos medicamentos. ${ }^{1}$ A proteína HSA é uma das mais abundantes no plasma humano, encontrada predominantemente nos fluídos corporais; possui importantes funções fisiológicas devido a sua alta capacidade como portadora e como reservatório para moléculas endógenas e exógenas. Apresenta-se como um monômero de $67 \mathrm{kDa}$; possui três domínios globulares, divididos em dois subdomínios: IA, IIA, IB, IIB, IIIA e IIIB. Os principais sítios de ligação para fármacos, referidos como sítio I e sítio II, estão localizados nas cavidades hidrofóbicas dos subdomínios IIA e IIIA, respectivamente, ${ }^{2-8}$ denominados de Sudlow I e II. A relevância em entendermos as bases estruturais da especificidade da interação entre a proteína HSA e moléculas consideradas como fármacos é devido ao fato de buscarmos e aperfeiçoarmos um melhor entendimento da interação, tanto ao nível atômico como molecular, que poderá definir então as possíveis abordagens terapêuticas para o tratamento de enfermidades e no alívio de sintomas advindos de doenças ou síndromes. ${ }^{9-12}$

A Simulação Computacional utilizada neste trabalho tem como propósito a busca de um maior entendimento das interações entre a proteína HSA e o aminoácido Dansilglicina (DanG), buscando subsídios teóricos para uma possível comparação com os resultados experimentais. Outra característica importante da simulação computacional, adotada neste trabalho, é o seu caráter preditivo das propriedades químicas e físicas de sistemas de interesse como, por

*e-mail: izabelle.tif@gmail.com exemplo, na predição da conformação adotada pela DanG no sítio de interação da HSA. ${ }^{13,14}$

A caracterização de como um fármaco pode se ligar na proteína HSA torna-se de suma importância na busca do entendimento de sua distribuição pelo corpo humano, na sua taxa de metabolismo e a sua excreção. Sendo assim, a caracterização, tanto do ponto de vista estrutural como de variações de energia de ligação, conduz a parâmetros que auxiliam na determinação da afinidade entre as espécies, da constante de ligação e do número de sítios de interação. Com este intuito, utilizamos o aminoácido DanG no estudo e caracterização dos sítios de ligações à proteína HSA, buscando uma explicação para a origem da sua quiralidade quando da formação do complexo entre ambos.

Aminoácidos dansilados apresentam um alto rendimento quântico de fluorescência; esse rendimento aumenta consideravelmente quando o aminoácido interage com os sítios da HSA. Observamos um deslocamento nos picos de emissão para comprimentos de onda menores do espectro eletromagnético devido à ocorrência de um fenômeno típico de transferência, do aminoácido, do meio hidrofílico para o meio hidrofóbico no interior da proteína. Com a diminuição da fluorescência do complexo HSA-DanG, provocado pela adição de um fármaco específico, podemos obter um parâmetro analítico para a detecção do seu sítio de ligação. ${ }^{15-20}$ Outra abordagem experimental que pode fornecer informações fundamentais sobre a interação entre as biomoléculas e os fármacos são os métodos espectrais de dicroísmo circular eletrônico, (ECD, Electronic Circular Dichroism). Além dos métodos experimentais de análise foram utilizados os métodos de simulação computacional baseados na Teoria do Funcional da Densidade (DFT) e no Docking Molecular na busca da interpretação dos dados obtidos experimentalmente e na elucidação da quiralidade induzida à molécula de DanG quando complexada à proteína HSA. ${ }^{21-27}$ 


\section{PARTE EXPERIMENTAL}

\section{Produtos químicos}

Dansilglicina (DanG - $\mathrm{C}_{14} \mathrm{H}_{16} \mathrm{~N}_{2} \mathrm{O}_{4} \mathrm{~S}$ ), Albumina do Soro Humano, livre de ácidos graxos e de globulinas, 99\% (HSA), Fosfato dibásico de sódio $\left(\mathrm{Na}_{2} \mathrm{HPO}_{4}\right)$, Fosfato monobásico de sódio $99 \%\left(\mathrm{NaH}_{2} \mathrm{PO}_{4}\right)$, Acetonitrila $99,8 \%\left(\mathrm{C}_{2} \mathrm{H}_{3} \mathrm{~N}\right)$, Tetrahidrofurano $99,5 \%$ (THF, C4H8O), Metanol 99,9\% $\left(\mathrm{CH}_{3} \mathrm{OH}\right)$, foram fornecidos pela Sigma Aldrich Chemical Co. (St. Louis, MO, EUA). Álcool etílico 99,66\% $\left(\mathrm{C}_{2} \mathrm{H}_{6} \mathrm{O}\right)$ fornecido pela J. T Baker Chemical Company (Phillipsburg, New Jersey). A solução estoque de HSA $1 \mathrm{mmol} \mathrm{L}^{-1}$ foi preparada dissolvendo-se a massa de 0,066 g de HSA em 1,0 mL de tampão fosfato de sódio $0,05 \mathrm{~mol} \mathrm{~L}^{-1}, \mathrm{pH}$ 7,0. 60,0 $\mu \mathrm{L}$ dessa solução foram diluídas para 2,0 mL, gerando uma concentração final de $30,0 \mu \mathrm{mol} \mathrm{L}^{-1}$, a qual foi submetida às análises. Para o preparo de $1,0 \mathrm{~mL}$ da dansilglicina (DanG) $10 \mathrm{mmol} \mathrm{L}^{-1}$ (Massa Molar $=308,35 \mathrm{~g} \mathrm{~mol}^{-1}$ ), foi pesado 0,00323 g de DanG e dissolvido em $1 \mathrm{~mL}$ de álcool etílico. A partir desta fizeram-se as diluições necessárias em água, metanol, etanol, acetonitrila e THF. A solução estoque foi mantida na geladeira e a solução de trabalho foi descartada após a medição. A concentração da solução estoque de HSA ( $\left.1 \mathrm{mmol} \mathrm{L}^{-1}\right)$, preparada em tampão fosfato $50 \mathrm{mmol} \mathrm{L}^{-1}, \mathrm{pH} \mathrm{7,0}$, foi determinada por sua absorbância na região do UV-Vis (HSA, $\varepsilon_{280 \mathrm{~mm}}=35.219 \mathrm{~mol}^{-1} \mathrm{~L} \mathrm{~cm}^{-1}$ ).

\section{Espectroscopia UV-Vis}

As medidas foram realizadas num espectrofotômetro UV-Vis Lambda 35 (Perkin Elmer, Shelton, CT, USA). O equipamento foi ajustado para determinar o espectro de absorbância no intervalo de 250-450 nm. O volume da solução, do aminoácido DanG,

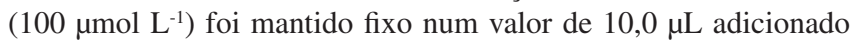
em cada solvente num volume fixo de 990,0 $\mu \mathrm{L}$, apresentando um volume final igual a 1.000,0 $\mu \mathrm{L}$. Antes de cada análise as amostras e a placa transparente com as amostras foram incubadas a temperatura ambiente por 5 minutos.

\section{Elipticidade: dicroísmo circular eletrônico (ECD)}

Os estudos de ECD foram realizados em um espectropolarímetro Jasco J-815 (Jasco, Japan) equipado com controle termostatizado e agitação magnética no compartimento da amostra. Todas as medidas foram corrigidas para a absorção do tampão fosfato $0,05 \mathrm{~mol} \mathrm{~L}^{-1}$, $\mathrm{pH}$ 7.0. Para os estudos na região do Ultra-Violeta próximo, foi utilizado um espectropolarímetro Jasco J-815 (Jasco, Japan). As medidas foram realizadas em uma cubeta de quartzo de $3 \mathrm{~mL}$ com $10 \mathrm{~mm}$ de caminho óptico, numa concentração de $30 \mu \mathrm{mol} \mathrm{L} \mathrm{L}^{-1}$ da proteína HSA e $30 \mu \mathrm{mol} \mathrm{L}{ }^{-1}$ do aminoácido DG. Os estudos foram feitos em tampão fosfato $0,05 \mathrm{M}, \mathrm{pH} 7.0$, à temperatura de $25^{\circ} \mathrm{C}$. Os espectros foram obtidos na faixa de comprimento de onda entre $250-500 \mathrm{~nm}$, com uma largura de banda de $1 \mathrm{~nm}$, com um tempo de resposta $1 \mathrm{~s}$, e tempo de varredura de $50 \mathrm{~nm} / \mathrm{min}$.

\section{Metodologia computacional}

\section{Teoria do Funcional da Densidade}

As conformações do aminoácido DanG em fase gasosa e nos solventes água, etanol, metanol, acetonitrila e THF foram calculadas ao nível DFT utilizando os funcionais híbridos B3LYP e CAM-B3LYP com o conjunto de funções de base 6-311++G(2d,p), usando o programa Gaussian $09^{28} \mathrm{e}$ as facilidades computacionais do GridUNESP. No cálculo dos espectros teóricos de ECD e de absorção UV-Vis foram utilizados os seguintes parâmetros no programa
Gaussian 09: TD(FULL,SINGLET,NSTATES=15) SCRF(SMD, SOLVENT). As estruturas otimizadas não apresentaram frequências imaginárias, não sendo, portanto, estados intermediários; foi utilizado: CAM B3LYP/6 311++G(2d,p) Opt Freq. A visualização e interpretação dos resultados foram feitas através do software GAUSSVIEWS 5.0. ${ }^{29}$

\section{Docking molecular}

O docking foi realizado utilizando as seguintes estruturas cristalográficas obtidas do banco de dados "Protein Data Bank" (PDB): $2 \mathrm{xvu}^{30}$ e $2 \mathrm{xw} 1^{30}$ com resolução de 2,6 e 2,5 Å, respectivamente. Estas estruturas são complexos cristalográficos: (a) 2xvu é uma HSA livre de ácidos graxos complexada com dansil- $L$-asparagina (DanN) em dois sítios, onde estão presentes os resíduos de aminoácidos W214 (sítio I) e Y411 (sítio II); (b) 2xvu é uma HSA livre de ácidos graxos complexada com dansil- $L$-norvalina (DanNV) em um único sítio, onde está presente o resíduo de aminoácido Y411 (sítio II).

Os cálculos de docking molecular foram realizados utilizando o software computacional baseado em algoritmos genéticos GOLD $(\mathrm{CCDC})^{31-33}$ v.5.6.2 e foi utilizada a função de ajuste GoldScore apresenta a seguir:

$$
\text { Função de ajuste }=S(\text { hb_ext })+1,3750 * S\left(v d w \_e x t\right)+S(\text { int })
$$

em que S(hb_ext) é a energia das ligações de hidrogênio proteína-ligante, S(vdw_ext) é a energia das interações de van der Waals proteína-ligante, e S(int) é uma penalidade para não incentivar as interações internas do ligante.

Os cálculos de docking foram realizados de maneira similar nos dois casos:

(a) foram adicionados átomos de hidrogênio em rotina interna do programa, e foram retiradas as moléculas de água, pois não foram observadas interações dos ligantes com as mesmas.

(b) os ligantes foram retirados e foi feito o redocking (reconstrução do complexo original) para avaliar os procedimentos e para podermos ter um score para ser comparado com os dos obtidos nos cálculos. Os raios das cavidades utilizadas para os cálculos (além da cavidade ocupada por cada um dos ligantes, conforme Figura 1), foram $\mathrm{R}=8 \AA$ em cada sítio para a proteína $2 \mathrm{xvu}$ e $\mathrm{R}=7 \AA$, no caso de $2 \mathrm{xv} 1$. A cavidade única em 2 xv1 ficou então com um raio calculado, $R_{\text {calc }}=10.146 \AA$ e origem $\mathrm{O}$ em: 9,35 ; 2,$43 ;-14,40$. No caso de 2 xvu uma das cavidades, que inclui o aminoácido W214, ficou com um $\mathrm{R}_{\text {calc }}=12,231 \AA$ e origem $\mathrm{O}$ em: 4,48; -9,22; 8,10; a outra cavidade, que inclui o aminoácido Y411, $R_{\text {calc }}=11,408 \AA$ e origem O em: 9,$12 ; 1,81 ;-15,38$.

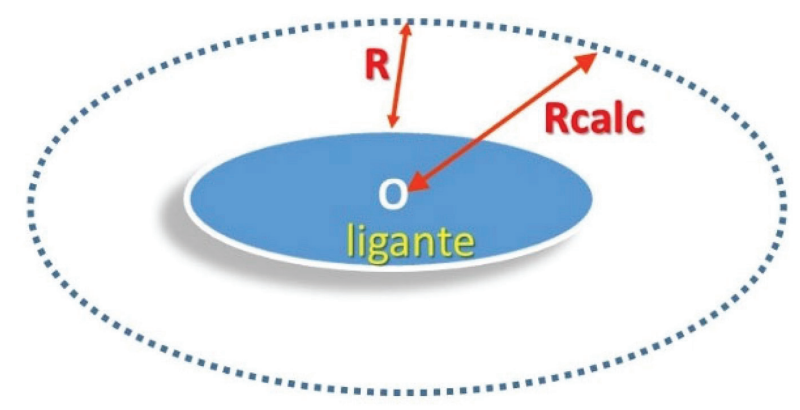

Figura 1. Esquema mostrando a cavidade que vai acomodar os ligantes no docking

Os ligantes para os cálculos de docking foram os seguintes:

(a) DanN: dansil- $L$-aparagina; o ligante do complexo cristalográfico na estrutura de código PDB igual a 2xvu, 
DanG1

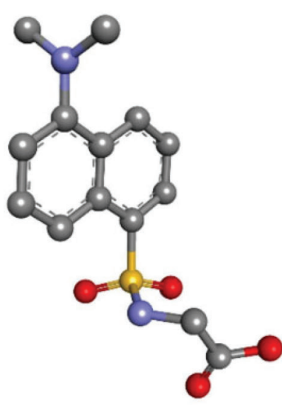

DanG2

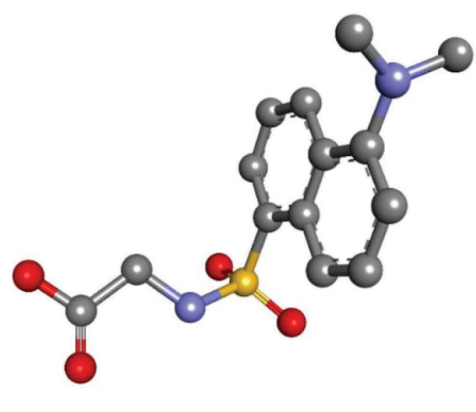

Sobreposição

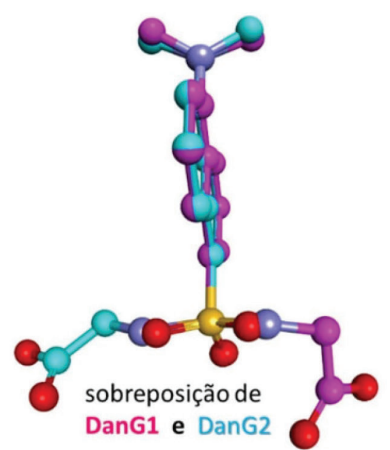

Figura 2. Ligantes utilizados no docking. DanG1: ângulo diedro C22-N15-C5-C4 = 103,31 ${ }^{\circ}$ e ângulo diedro N29-S26-C9-C3 = -57,03 ${ }^{\circ}$ : DanG2: ângulo diedro C22-N15-C5-C4 = -90,01 e ângulo N29-S26-C9-C3 = -70,01. A numeração segue aquela apresentada na Figura 5

(b) DanNV: dansil- L-norvalina: o ligante do complexo cristalográfico na estrutura de código PDB igual a exv1,

(c) DanG1: dansil-glicina (Figura 2), obtida por cálculos ab initio apresentados neste traballho e

(d) DanG2: dansil-glicina (Figura 2) obtida da base de dados PubChem (Open Chemistry Database), https://pubchem.ncbi.nlm. nih.gov/compound/Dansylglycine.

Para os cálculos de docking somente os ligantes foram considerados flexíveis, ou seja, sem restrições aos ângulos de torção. A seleção dos compostos resultantes dos cálculos de docking foi feita em programas de visualizaçao gráfica (Accelrys DS Visualizer v3.5, Biovia Discovery Studio 2017 R2) 34 $^{34}$ considerando-se o padrão de poses (posição, conformação e orientação) do ligante e para aqueles dentro do padrão, com maior score.

Após a seleção da melhor pose, foram consideradas as interações não covalentes dos ligantes, dentro do sítio e analisadas quanto ao tipo e característica da interação, utilizando programas de visualização gráfica. ${ }^{34,35}$ Também foram realizados cálculos para avaliar o gradiente reduzido da densidade eletrônica (RDG) ${ }^{36-39}$ a partir dos quais foi possível visualizar as isosuperfícies RDG das interações não covalentes (NCI).

\section{RESULTADOS E DISCUSSÃO}

\section{Estudo do espectro de absorbância da DanG em diversos solventes}

Na Figura 3(a) apresentamos os espectros de absorção na região do UV-Vis do aminoácido DanG nos diversos solventes considerados onde não foi observada uma variação expressiva nos máximos de absorção. Uma exceção foi com o solvente água, na qual o espectro apresentou-se menos intenso e com uma menor definição entre as bandas de absorção no valor de $324 \mathrm{~nm}$. Os outros solventes apresentaram um máximo de absorção (Tabela 1) identificada como uma transição $\mathrm{n} \rightarrow \pi^{*}$, e no caso do solvente metanol esse valor foi igual a $338 \mathrm{~nm}$, e de $338 \mathrm{~nm}$ para o etanol, de $343 \mathrm{~nm}$ para acetonitrila e de $340 \mathrm{~nm}$ para o solvente THF. Os diversos solventes foram utilizados pelo fato de terem caracteríticas próticas e apróticas, como constatado por seus valores de constante dielétrica (Tabela 1), onde buscamos identificar uma possível modificação no espectro na regição do UV-Vis e relacionar com uma possível interpretação para o fato de o aminoácido DanG ser transferido de um meio hidrofílico para um meio hidrofóbico quando da interação com a proteína HSA. No solvente água observamos um deslocamento hipsocrômico (para comprimentos de onda menores do espectro eletromagnético) junto com um efeito hipocrômico (uma diminuição da absorbância) na região de $250 \mathrm{~nm}$, diferentemente dos demais solventes utilizados onde não observamos modificações tanto nas posições como nas intensidades do espectro de absorção. Na Figura 3(b) observamos uma pequena diminuição no valor da absorbância para os solventes: água, etanol e acetonitrila. No entanto, não houve qualquer deslocamento no máximo de absorção do aminoácido DanG quando comparado com os resultados experimentais. Este comportamento deverá ser mais bem analisado, pois no modelo teórico consideramos o solvente de maneira implícita, levando em consideração somente o valor da constante dielétrica dos mesmos.

Para a determinação dos espectros de absorção do aminoácido DanG na região do UV-Vis e ECD utilizamos o funcional CAM-B3LYP e a função de base 6-311++G(2d, p), nos mesmos solventes..$^{40-42} \mathrm{Na}$ Figura 3(b) apresentamos os espectros obtidos para o aminoácido DanG nos diversos solventes. Podemos observar uma boa concordância entre os valores obtidos na simulação computacional e aqueles obtidos experimentalmente, ressaltando o caso do solvente água que apresentou um deslocamento em relação, ao espectro obtido teóricamente, para a região do azul do espectro eletromagnético. $\mathrm{Na}$ Tabela 1 apresentamos o valor da constante dielétrica para os solventes estudados bem como o valor para o máximo de absorção no espectro de UV-Vis.

Tabela 1. Máximo de absorção e constante dielétrica $\varepsilon$ dos solventes utilizados

\begin{tabular}{lcc}
\hline Solventes & $\varepsilon$ & Máximo de absorção $(\mathrm{nm})$ \\
\hline Água & 80,00 & 324 \\
Metanol & 24,30 & 338 \\
Etanol & 33,00 & 338 \\
Acetonitrila & 36,60 & 343 \\
THF & 7,52 & 340
\end{tabular}

Interação do aminoácido DanG com a proteína HSA: dicroísmo circular eletrônico (ECD)

De posse do conhecimento de que o aminoácido DanG liga-se ao sítio II da proteína HSA, ${ }^{26}$ utilizamos a técnica de ECD para descobrirmos em qual configuração, $R$ ou $S$, a mesma se apresenta quando complexada com a HSA. Observamos que a molécula de DanG, quando diluída em tampão no $\mathrm{pH} 7,0$, não apresentou sinal de dicroísmo circular, o que já era previsto, pois não se trata de uma molécula opticamente ativa, isto é, não possui átomos de carbono quirais. Na Figura 4(a) o espectro de ECD foi medido na faixa de $250 \mathrm{~nm}$ a $500 \mathrm{~nm}$ e observamos que a complexação entre DanG e 

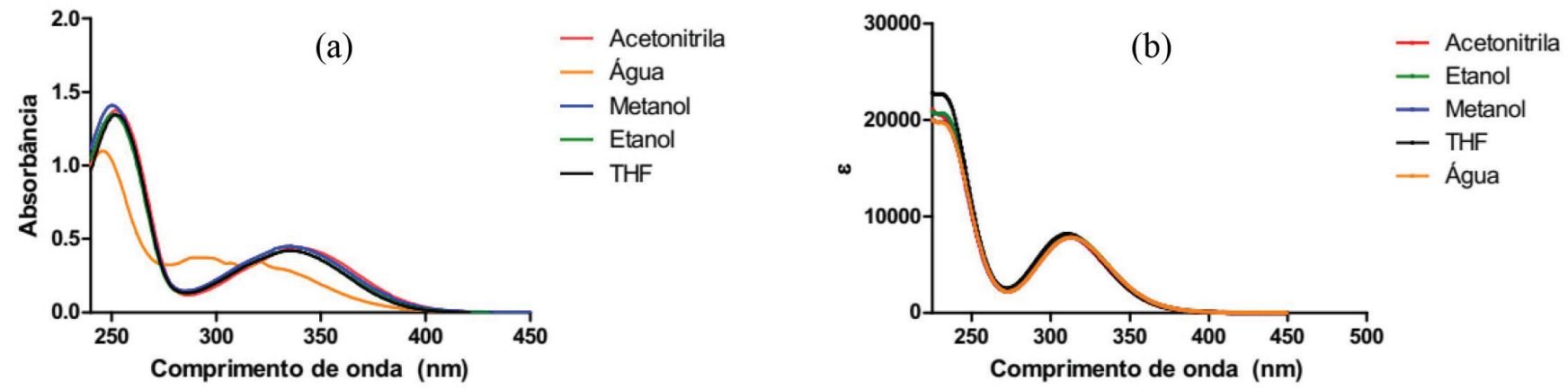

Figura 3. (a) Espectros de absorção experimental da DanG, $100 \mu \mathrm{mol} L^{-1}$ em diversos solventes: água; metanol; etanol; acetonitrila e THF (b) Espectros de absorção teórico da DanG nos mesmos solventes

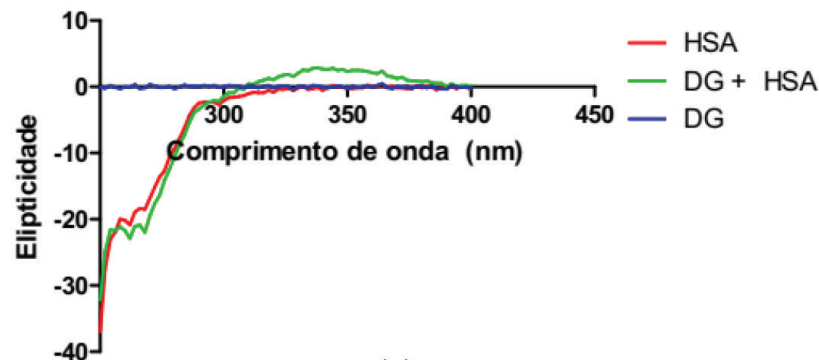

(a)

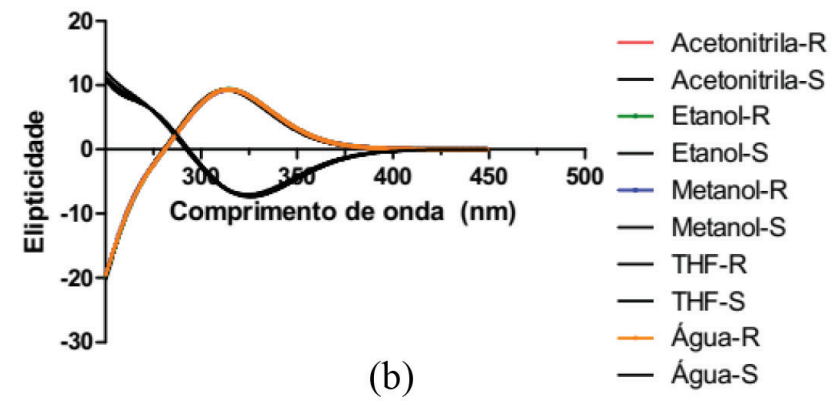

(b)

Figura 4. (a) Complexação da DanG-HSA (b) ECD da DanG em diferentes solventes e suas configurações R e S

proteína HSA resultou no aparecimento de um sinal de ICD centrado em 346 nm. Esta indução de quiralidade na molécula de DanG é devido à sua interação com a proteína, e pode ser explicada pela ligação ao ambiente assimétrico que caracteriza os sítios de ligação nas proteínas.

Foi utilizado o funcional híbrido B3LYP e o conjunto de base 6-311++G(2d, p) para o estudo teório do ECD, ${ }^{43,44}$ da DanG nas configurações $R$ e $S$. Na Figura 4(b) observamos que, na presença dos diferentes solventes, a molécula de DanG apresentou configuração $R$ com sinal positivo, e na configuração $S$ um sinal negativo no sinal de ECD. A comparação entre o espectro de ECD experimental com aqueles simulados em diferentes solventes nos leva a concluir que a conformação adquirida pelo aminoácido DanG ao se ligar à proteína deve apresentar uma configuração $R$.

\section{Estudo da superfície de energia potencial (SEP) e ECD da DanG}

Na Figura 5 apresentamos a estrutura da molécula de DanG com a numeração utilizada para a identificação dos átomos a serem considerados nos cálculos da SEP e de ECD referentes aos ângulos diedros.

A SEP da DanG foi calculada a partir do funcional híbrido B3LYP e a função de base 6-31G(2d, p) no vácuo. Na Figura 6 apresentamos a SEP (com intervalos de $10^{\circ}$ entre cada ponto da curva desde $0^{\circ}$ até $360^{\circ}$ ), na qual podemos verificar a presença de mínimos e máximos de energia como uma função do ângulo diedro representado pelos átomos: C22-N17-C5-C4. A SEP foi calculada de maneira não rígida, isso é, para cada valor do ângulo diedro a estrutura da molécula inteira foi otimizada.

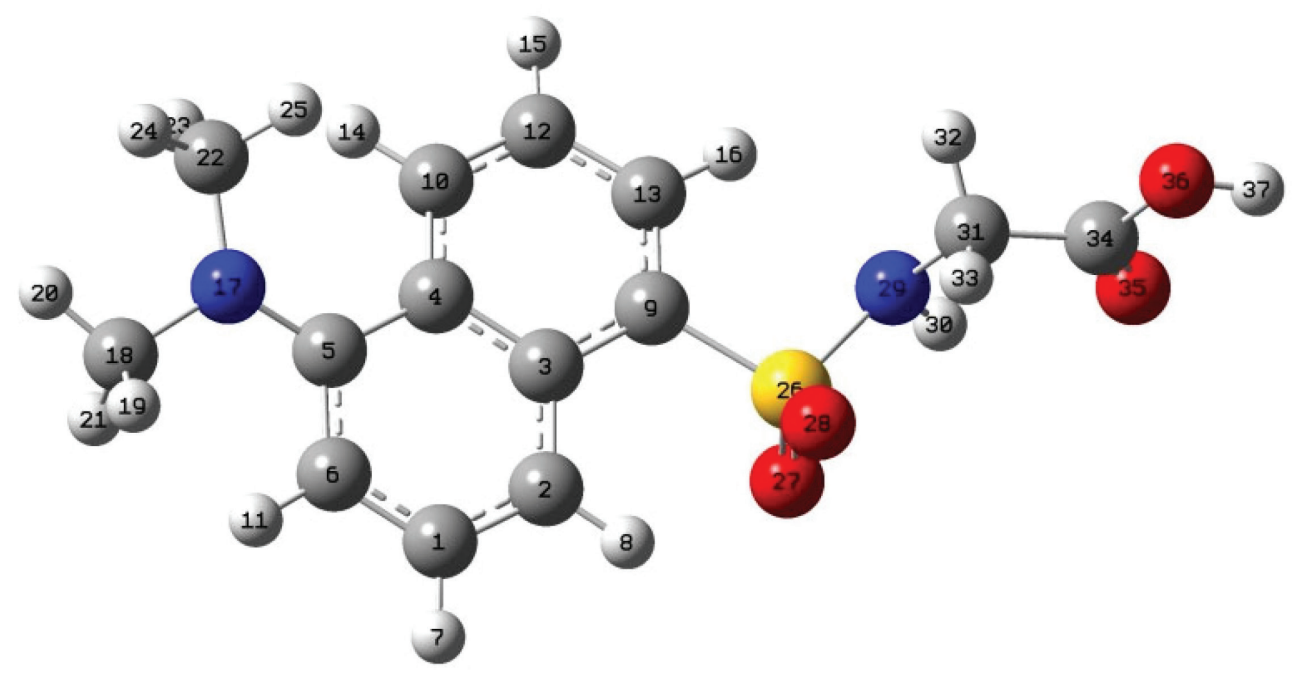

Figura 5. Estrutura da DanG com os átomos numerados 

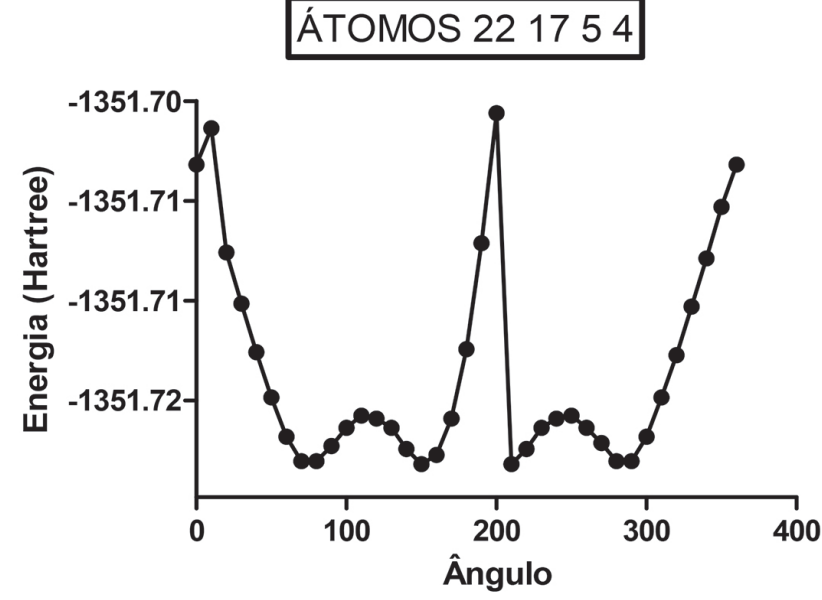

Figura 6. SEP do aminoácido DanG

Apresentamos na Figura 7 (a) o espectro da DanG no solvente água para os ângulos diedros de $0^{\circ}, 10^{\circ}, 120^{\circ}, 130^{\circ}, 140^{\circ}$ e $150^{\circ}$ onde podemos verificar que o sinal de ECD obtido foi positivo para todos os ângulos diedros considerados. Para o ângulo de $0^{\circ} \mathrm{o}$ máximo no espectro de ECD encontra-se em $350 \mathrm{~nm}$, e à medida que aumentamos o ângulo diedro este máximo sofre um descolamento para comprimentos de onda menores: para o ângulo de $130^{\circ}$ o valor máximo é igual a 310 nm e à medida que aumentamos o diedro o ângulo para $140^{\circ}$ e $150^{\circ}$ o valor máximo passa a ser igual 315 e $320 \mathrm{~nm}$, respectivamente. $\mathrm{Na}$ Figura 7 (b) apresentamos o espectro da DanG no solvente água para os ângulos diedros de $20^{\circ}, 30^{\circ}, 40^{\circ}, 50^{\circ}, 60^{\circ}, 70^{\circ}$ e $80^{\circ}$, a partir da qual podemos verificar que o sinal de ECD obtido foi negativo para todos os ângulos diedros considerados. À medida que aumentamos o valor do ângulo diedro no intervalo de $20^{\circ}$ a $60^{\circ}$, o sinal de ECD não sofre mudanças significativas situando-se próximo a $350 \mathrm{~nm}$. À medida que aumentos o valor do ângulo diedro para $70^{\circ}$ e $80^{\circ}$, foi observado um aumento na intensidade do sinal e um deslocamento hipsocrômico, com máximos de elipcitidade iguais a 326 e $317 \mathrm{~nm}$, respectivamente.
Nos cálculos da SEP da DanG, considerando os ângulos diedros formados pela ligação do grupo $-\mathrm{N}\left(\mathrm{CH}_{3}\right)_{2}$ do anel naftalenico, mostraram que a partir das várias conformações possíveis e seus respectivos ECDs teóricos o valor do ângulo diedro igual a $150^{\circ}$ (ECD positivo centrado em $320 \mathrm{~nm}$ ) apreentaram uma boa concordância com o espectro obtido experimental. Por outro lado, o diedro de $80^{\circ}$ apresentou um sinal do espectro de ECD oposto ao observado experimentalmente.

Com o objetivo de obter e confirmar os resultados de ECD obtidos tanto teórica como experimentalmente, foi realizado cálculos de docking molecular. Esperávamos que os resultados de docking molecular para a estrutura da molécula de DanG, no sítio de interação, apresentasse um valor de ângulo diedro próximo àquele obtido pela técnica de DFT e confirmasse o sinal de ECD obtido experimentalmente.

Os resultados obtidos para os cálculos de docking são apresentados na Tabela 2, na qual estão inseridos os valores de energia para a melhor pose (posição, orientação e conformação do ligante) na cavidade da proteína HSA em cada cálculo realizado. Foram realizadas três simulações: (a) uma para ligantes no sítio receptor de 2xw1; nesse sítio está presente o resíduo de aminoácido Tyr411; (b) uma para ligantes no sítio receptor de $2 \mathrm{xvu}$; nesse sítio está presente o resíduo de aminoácido Trp214; (c) uma para ligantes no sítio receptor de $2 \mathrm{xvu}$; nesse sítio está presente o resíduo de aminoácido Tyr411.

Os dados apresentados na Tabela 2 mostram que, para o caso em que o receptor é a proteína 2xw1, em que apenas o sítio II está ocupado pelo ligante, o escore do redocking (reconstrução do complexo cristalográfico) e os de DanG são muito próximos, indicando que o ligante DanG pode se acomodar neste sítio assim como o ligante cristalográfico.

Para o receptor de $2 \mathrm{xvu}$, oberva-se que os cálculos de redocking indicam uma preferência pelo sítio II, sugerindo que este sítio é o preferencial. Os ângulos de torsão das moléculas são apresentados na Tabela 3.

De acordo com os resultados apresentados na Tabela 3, podemos observar que apesar das moléculas ligantes DanG terem condições iniciais diferentes, a condição final, obtida com o docking molecular á praticamente a mesma.
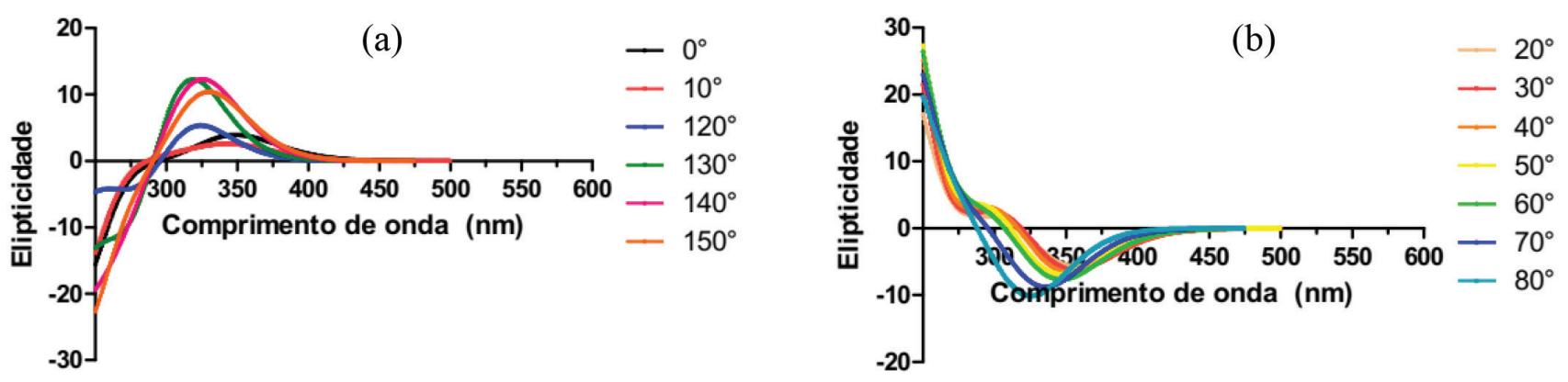

Figura 7. Espectros de dicroismo circular (a) positivo e (b) negativo da DanG no solvente água em função dos ângulos diedros

Tabela 2. Resultados de docking molecular para 2xvu e 2xw1

\begin{tabular}{|c|c|c|c|c|}
\hline Proteína & Ligante & $\begin{array}{c}\text { Sítio I (W214) } \\
\text { Energia (kcal/mol) }\end{array}$ & $\begin{array}{c}\text { Sítio II (Y411) } \\
\text { Energia }(\mathrm{kcal} / \mathrm{mol})\end{array}$ & $\begin{array}{c}\Delta \text { Escore }=\text { Sítio II }- \text { Sítio I } \\
(\mathrm{kcal} / \mathrm{mol})\end{array}$ \\
\hline \multirow{4}{*}{$2 x v u$} & DanN-1 & 70,7 & 87,2 & 16,3 \\
\hline & DanN-2 & 73,4 & 86,7 & 13,3 \\
\hline & DanG1 & 62,7 & 77,8 & 15,1 \\
\hline & DanG2 & 61,3 & 78,3 & 17,0 \\
\hline \multirow{3}{*}{ 2xw1 } & DanNV & - & 60,9 & - \\
\hline & DanG1 & - & 50,7 & - \\
\hline & DanG2 & - & 61,8 & - \\
\hline
\end{tabular}


Tabela 3. Ângulos de torção de DanG para resultados de docking no sítio II

\begin{tabular}{lccc}
\hline Condições & Ligante & $\begin{array}{c}\text { angulo }\left({ }^{\circ}\right) \\
\text { C22-N17-C5-C4 }\end{array}$ & $\begin{array}{c}\text { ângulo }\left({ }^{\circ}\right) \\
\text { N29-S26-C9-C3 }\end{array}$ \\
\hline \multirow{2}{*}{ Inicial } & DanG1 & 103,31 & MD-57,03 \\
& DanG2 & $-90,01$ & 70,01 \\
\hline \multirow{2}{*}{ Final } & DanG1 & 111,96 & $-61,69$ \\
& DanG2 & 110,90 & $-68,28$ \\
\hline
\end{tabular}

Na Figura 8 apresentamos o ligante DanG no sítio II da proteína HSA em uma representação de superfície para a proteína, a partir da qual podemos observar que o grupo naftil ocupa o bolso interno da proteína e o grupo carboxílico está voltado para o solvente.

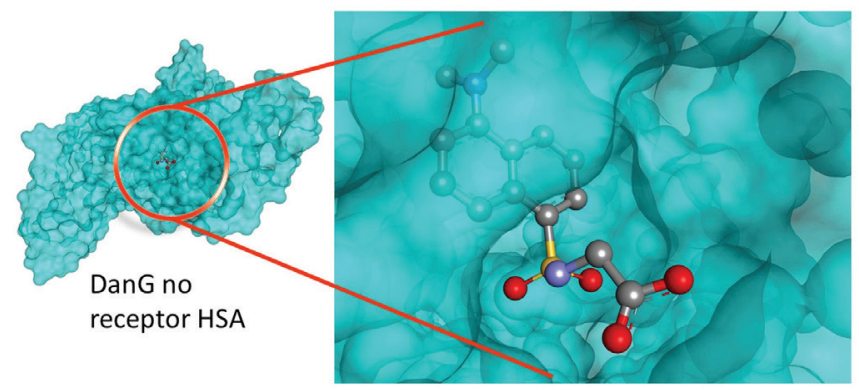

Figura 8. Molécula de DanG no sítio II da proteína HSA

A análise das interações realizada com o programa de visualização gráfica ${ }^{36}$ indica que o grupo naftil da molécula se estabiliza pelas interações do tipo $\pi$ entre os aminoácidos Asn391 e Leu453, conforme pode ser obervado na Figura 9. O grupo carboxílico da DanG faz ligações de hidrogênio com Arg410 e o grupo sulfona faz interações com a Tyr411.

A Figura 9 mostra que:

(a) Os principais aminoácidos que interagem com a molécula DanG no sítio II são Tyr411, Leu453, Asn391 e Arg410,

(b) B1: o grupo naftil fica estabilizado pelas interações $\mathrm{CH} \cdots \pi$ com o resíduo de Leu453 e com o resíduo de aminoácido Asn391.

B2: É apresentada a isosuperfície NCI que foi gerada usando densidades moleculares para $\mathrm{RDG}=0.30$ u.a. entre o grupo naftil e Leu453 B3: É apresentada a isosuperfície NCI entre o grupo naftil e Asn391.

(c) São apresentadas as ligações de hidrogênio (C1) entre a Arg410 e o grupo carboxílico de DanG e em C2 as isosuperfícies NCI para as ligações de hidrogênio entre a Arg410 e o grupo carboxílico de DanG.

(d) São apresentadas as interações (D1) entre a Tyr411 e o oxigênio do grupo sulfona da DanG e em D2 as isosuperfícies NCI para as interações entre a Tyr411 e o oxigênio do grupo sulfona da DanG.

A partir da configuração obtida para a molécula de DanG nos cálculos de docking molecular, obtivemos os espectros de UV-Vis e de ECD da molécula de DanG a partir da simulação computacional utilizando o funcional CAM-B3LYP e a função de base $6-311++G(3 d f, 2 p)$. Na Figura 10 apresentamos os especros de UV-Vis (a) e ECD (b) representados como a força do oscilador e o poder rotatório, respectivamente. Podemos observar que ocorre uma boa concordãncia entre os resultados do docking molecular e aqueles obtidos através da técnica DFT e dos resultados experimentais.

\section{Estudo dos orbitais moleculares HOMO-LUMO da DanG}

Na Figura 11 (a) apresentamos os orbitais moleculares HOMO e LUMO para a molécula de DanG para o ângulo diedro, formado entre os átomos C22-N17-C5-C4, igual a 80. O orbital HOMO (de número 81) e o LUMO (de número 82) são os responsáveis pela transição eletrônica observada no espectro de ECD, obtida no nível de teoria DFT com o funcional híbrido CAM-B3LYP e a função de base 6-311++G(2d, p) no vácuo. A transição HOMO-LUMO se refere à passagem de um elétron localizado no par de elétrons $n$ do átomo de Nitrogênio N17 para um orbital $p$ localizado no átomo de Carbono C5. Essa transição está relacionada ao sinal negativo no espectro de ECD apresentado na Figura 7 (b) localizado em 317 nm.

Na Figura 11 (b) apresentamos os orbitais moleculares HOMO e LUMO para a molécula de DanG para o ângulo diedro C22-N17C5-C4 igual a $150^{\circ}$. O orbital HOMO (de número 81) e o LUMO (de número 82) são os responsáveis pela transição eletrônica observada
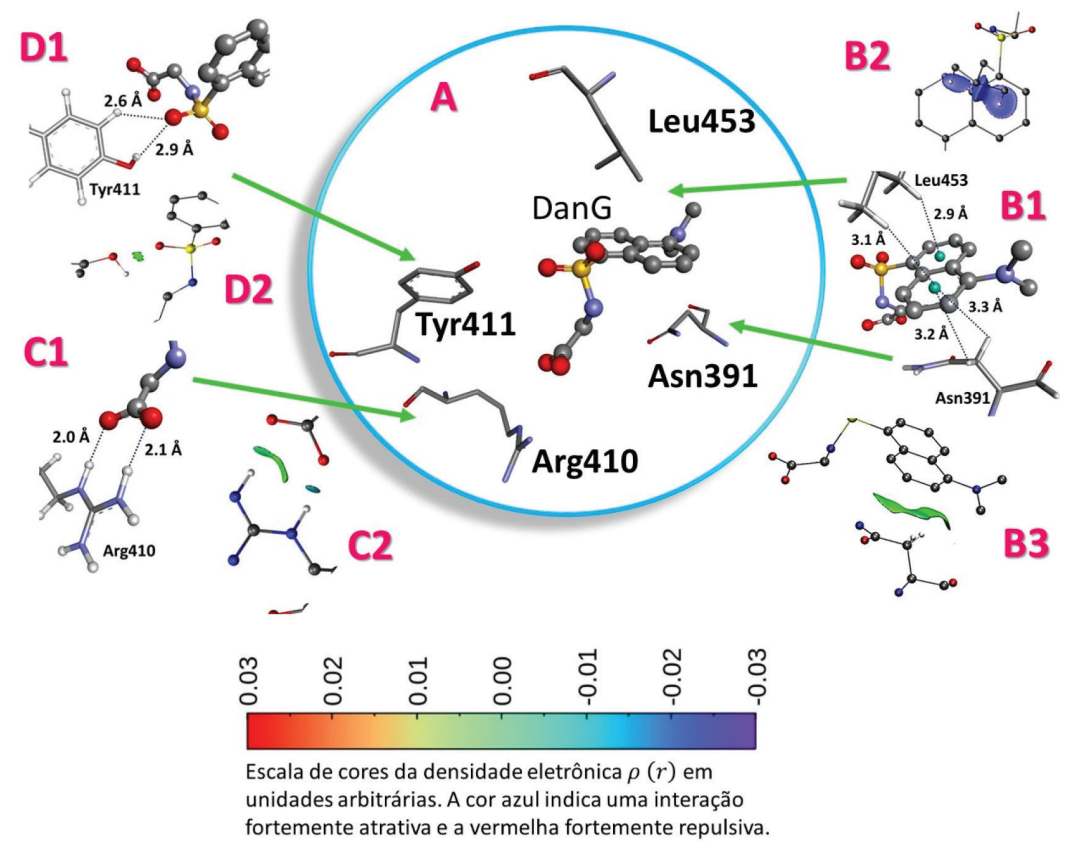

Figura 9. Interações entre DanG no sítio II da HSA 

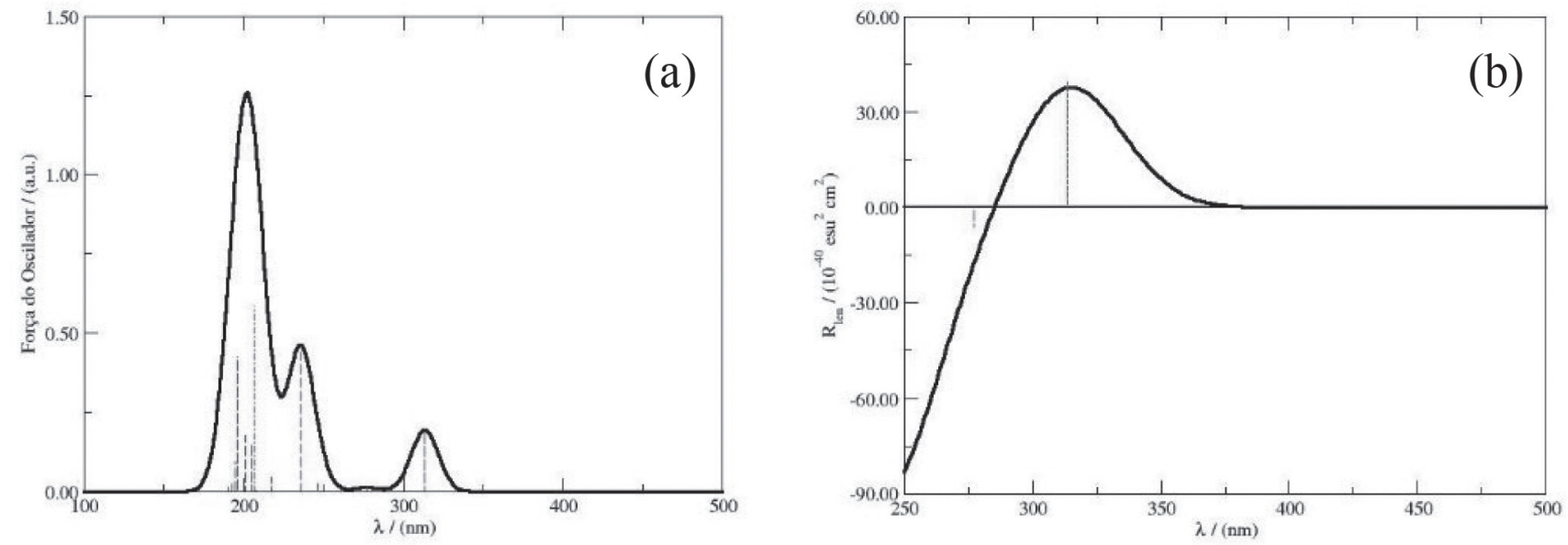

Figura 10. Espectros eletrônicos de (a) UV-Vis e (b) Dicroísmo Circular da DanG obtidos em etanol
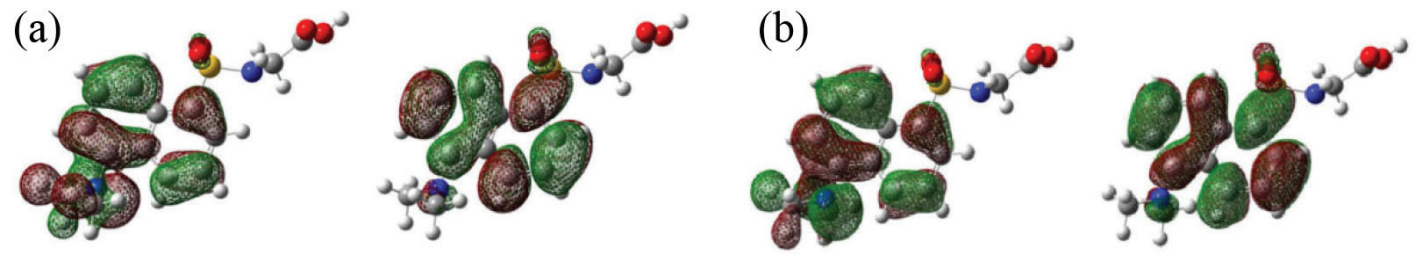

Figura 11. Principais orbitais moleculares (a) HOMO e LUMO da DanG para o diedro de 80 $0^{\circ}$ (b) HOMO e LUMO da DG para o diedro de $150^{\circ}$

no espectro de ECD, obtida no nível de teoria DFT com o funcional híbrido CAM-B3LYP e a função de base 6-311++G(2d, p) no vácuo. A transição HOMO-LUMO se refere à passagem de um elétron localizado no par de elétrons $n$ do átomo N17 para um orbital $p$ localizado no átomo C5. Esta transição está relacionada ao sinal positivo no espectro de ECD apresentado na Figura 5 (a) localizado em 333 nm.

As contribuições dos orbitais atômicos da DanG para os orbitais moleculares HOMO e LUMO em função do ângulo diedro foram obtidas a partir da análise dos orbitais moleculares e foram escolhidos aqueles que são predominantes para a formação do orbital molecular que descrevem as transições eletrônicas.

Podemos verificar que para o ângulo diedro igual a $80^{\circ}$ a maior contribuição para o orbital molecular HOMO está relacionada ao átomo C5 com o orbital atômico 5s e para o LUMO a maior contribuição está relacionada ao átomo de Carbono (4) com o orbital atômico $5 \mathrm{~s}$.

Para o ângulo diedro igual a $150^{\circ}$, a contribuição para o HOMO e LUMO está associada aos átomos de N17 com o orbital 4pz e o átomo de C5 com o orbital 5pz.

\section{CONCLUSÕES}

Os resultados obtidos mostraram que a DanG nos solventes: água, metanol, etanol, acetonitrila e THF, apresenta um comprimento de onda máximo de absorção em aproximadamente em 340 $\mathrm{nm}$, indicando uma transição $\mathrm{n} \rightarrow \pi^{*}$. Além disso, o uso desses dados experimentais foi útil para relacionarmos com os espectros teóricos, em que observamos que os espectros com as configurações $R$ e $S$ não apresentaram mudanças significativas em relação ao comprimento de onda máximo. Com os dados de ECD, pudemos demonstrar que a complexação de HSA com DanG leva ao aparecimento de uma banda ECD positiva centrada em $346 \mathrm{~nm}$. Esse fenômeno pode ser explicado pelo aparecimento da quiralidade na DanG quando induzida pelo meio, no presente caso o sítio II da proteína HSA. A comparação entre o espectro de ECD experimental com aqueles obtidos por DFT, em diferentes solventes, nos leva a concluir que a conformação adquirida pela DanG ao se ligar à proteína se assemelha a configuração $\mathrm{R}$.
Cálculos da superfície de energia potencial (SEP) da DanG nos mostram que os grupos envolvidos no fenômeno do aparecimento do sinal de ECD está relacionado ao grupo $-\mathrm{N}\left(\mathrm{CH}_{3}\right)_{2}$ do anel naftalenico. A análise das várias conformações obtidas e seus respectivos ECDs teóricos revelaram que o diedro de $150^{\circ}$ (ECD positivo centrado em $320 \mathrm{~nm}$ ) apresentou excelente similaridade com o espectro experimental. Por outro lado, o diedro de $80^{\circ}$ apresentou um sinal do espectro de ECD oposto ao observado experimentalmente. Além disso, observamos que o átomo de nitrogênio do grupo $-\mathrm{N}\left(\mathrm{CH}_{3}\right)_{2}$ apresentou a maior contribuição para a transição HOMO-LUMO que dá origem à transição eletrônica $n \rightarrow \pi^{*}$ envolvida na geração do sinal de ECD.

Os resultados dos cálculos de docking realizados em proteína livre de ácidos graxos com compostos dansilados no sítio 2 (onde está o aminoácido Tyr411) mostram que o composto DanG tem possibilidade similar de se ligar a esse sítio da mesma forma que o ligante cristalográfico DanNV, para a estrutura de código pdb 2xw1. Os estudos de docking foram realizados em duas situações no caso do receptor de código pdb 2xvu e o ligante DanG poderia ocupar o sítio I e/ou o sítio II do receptor. Observou-se que DanG liga-se preferencialmente ao sítio II com uma diferença de escore de mais de $15 \mathrm{kcal} \mathrm{mol}^{-1}$ em favor deste.

As análises de docking molecular mostraram que no sítio II o grupo naftil está estabilizado, dos dois lados, por interações do tipo $\mathrm{CH} \cdots \pi$ entre os aminoácidos Leu453 e Asn391, ficando praticamente imobilizado por estas interações. O grupo carboxílico e o átomo de oxigênio do grupo sulfona do ligante DanG fica estabilizado por interações com Arg410 e Tyr411, respectivamente. Os cálculos de isosuperfície de densidade eletrônica mostram que estas interações são atrativas.

As análises de docking molecular mostraram também que a complexação entre DanG e a proteína HSA gerou uma conformação com um diedro igual $\mathrm{a} 100^{\circ}$, muito similar àquele obtido teoricamente (DFT) e cujo ECD está em acordo com o resultado experimental. Em conclusão, o estudo das possíveis conformações para a dansilglicina e o cálculo do seu ECD teórico permitiram identificar a origem do sinal de ECD obtido pela complexação entre DanG e HSA. 


\section{AGRADECIMENTOS}

Este trabalho foi financiado pela Fundação de Amparo à Pesquisa do Estado de São Paulo (FAPESP, auxílios: 2016/205495, 2015/22338-9, 308480/2016-3 e INCT.Bio.Nat 2014/50926-0), Conselho Nacional de Desenvolvimento Científico e Tecnológico (CNPq: 302793/2016-0, 306975/2013-0, IC:440503/2014-0, IC:308480/2016-3 e 305541/2017-0) e CAPES. Esta pesquisa tornou-se possível graças aos recursos computacionais disponibilizados pelo Núcleo de Computação Científica (NCC/GridUNESP) da Universidade Estadual Paulista (UNESP).

\section{REFERÊNCIAS}

1. Lindup, W. E.; Orme, M. C.; Br. Med. J. 1981, 282, 212.

2. Fanali G.; Di Masi A.; Trezza, V.; Marino, M.; Fasano, M.; Mol. Aspects Med. 2012, 33, 209.

3. Sudlow, G.; Birkett, D. J.; Wade, D. N.; Mol. Pharmacol. 1976, 12, 1052 .

4. Sudlow, G.; Birkett, D. J.; Wade, D. N.; Mol. Pharmacol. 1975, 11, 824.

5. Kragh-Hansen, U.; Chuang, V. T. G.; Otagiri, M.; Biol. Pharm. Bull. 2002, 25, 695 .

6. Gundry, R. L.; Proteomics: Clin. Appl. 2007, 1, 73.

7. Peters, T.; All about Albumin: Biochemistry, Genetics, and Medical Applications, $1^{\text {st }}$ ed., Academic Press, Inc: San Diego, 1996.

8. Graham, A.; Li, G.; Chenm, Y.; Morgan, J.; Oseroff, A.; Dougherty, T. J.; Pandey, R. K. Photochem. Photobiol. 2003, 77, 561.

9. Chaves, O.; Echevarria, Á.; Esteves-Souza, A.; Maciel, M.; Netto-Ferreira, J. C.; J. Braz. Chem. Soc. 2018, 29, 1786.

10. Chaves, O. A.; Santos, M. R. L.; Santánna, C. M.; Echevarria, A.; Ferreira, A. B. B.; Netto-Ferreira, J. C.; Mediterr. J. Chem. 2018, 7, 8.

11. Lakowicz, J. R.; Principles of Fluorescence Spectroscopy. $3^{\text {rd }}$ ed., Springer: Boston, 2006.

12. Ximenes, V. F.; Morgon, N. H.; Souza, A. R.; Chirality 2018, 31, 1.

13. Souza, A.R.; Morgon, N. H.; Rev. Virtual Quim. 2016, 8, 417.

14. Petitpas, I.; Bhattacharya, A. A.; Twine, S.; East, M.; Curry, S.; J. Biol. Chem. 2001, 276, 22804.

15. Bertucci, C.; Domenici, E. C.; Med. Chem. 2002, 9, 1463.

16. Bhattacharya, A. A.; Curry, S.; Franks, N. P.; J. Biol. Chem. 2000, 275, 38731.

17. Ryan, A. J.; Ghuman, J.; Zunszain, P. A.; Chung, C. W.; Curry, S.; J. Struct. Biol 2011, 174, 84.

18. Simard, J. R.; Zunszain, P. A.; Hamilton, J. A.; Curry, S.; J. Mol. Biol. 2006, 361, 336.

19. Bertucci, C.; Pistolozzi, M.; Simone, A. D.; Anal. Bioanal. Chem. 2010, $398,155$.

20. Ali J. R.; Ghuman, J.; Zunszain, P. A.; Chung, C. W.; Curry, S.; J. Struct. Biol. 2011, 174, 84 .

21. Chatterjee, T.; Pal, A.; Dey, S.; Chatterjee, B. K.; Chakrabarti, P.; PLoS One 2012, 7, e37468.

22. Bertucci, C.; Pistolozzi, M.; Chirality 2008, $20,552$.
23. Koch, W.; Holthausen, M. C.; A Chemist's Guide to Density Functional Theory, $2^{\text {nd }}$ ed., Wiley-VCH: Weinheim, 2001.

24. Allenmark, S.; Chirality 2003, 15, 409.

25. Junior, N. N.; Tese de Doutorado, Universidade de São Paulo, Brasil, 2013.

26. Graciani, F. S.; Ximenes, V. F.; PLoS One 2013, 8, 1.

27. Arruda, P. M.; Dissertação de Mestrado, Universidade Federal do Espírito Santo, Brasil, 2009.

28. Frisch, M. J.; Trucks, G. W.; Schlegel, H. B.; Scuseria, G. E.; Robb, M. A.; Cheeseman, J. R.; Scalmani, G.; Barone, V.; Petersson, G. A.; Nakatsuji, H.; Li, X.; Caricato, M.; Marenich, A. V.; Bloino, J.; Janesko, B. G.; Gomperts, R.; Mennucci, B.; Hratchian, H. P.; Ortiz, J. V.; Izmaylov, A. F.; Sonnenberg, J. L.; Williams-Young, D.; Ding, F.; Lipparini, F.; Egidi, F.; Goings, J.; Peng, B.; Petrone, A.; Henderson, T.; Ranasinghe, D.; Zakrzewski, V. G.; Gao, J.; Rega, N.; Zheng, G.; Liang, W.; Hada, M.; Ehara, M.; Toyota, K.; Fukuda, R.; Hasegawa, J.; Ishida, M.; Nakajima, T.; Honda, Y.; Kitao, O.; Nakai, H.; Vreven, T.; Throssell, K.; Montgomery, J. A., Jr.; Peralta, J. E.; Ogliaro, F.; Bearpark, M. J.; Heyd, J. J.; Brothers, E. N.; Kudin, K. N.; Staroverov, V. N.; Keith, T. A.; Kobayashi, R.; Normand, J.; Raghavachari, K.; Rendell, A. P.; Burant, J. C.; Iyengar, S. S.; Tomasi, J.; Cossi, M.; Millam, J. M.; Klene, M.; Adamo, C.; Cammi, R.; Ochterski, J. W.; Martin, R. L.; Morokuma, K.; Farkas, O.; Foresman, J. B.; Fox, D. J.; Gaussian 09, Revision D.01; Gaussian Inc., Wallingford CT, 2013.

29. Foresman, J. B.; Frisch, E.; Exploring chemistry with electronic structure methods, $2^{\text {nd }}$ ed., Gaussian Inc., Pittsburg, 1996.

30. Chaves, O. A.; Tavares, M. T.; Cunha, M. R.; Parise, R. F.; Santánna, C. M.; Netto-Ferreira, J. C.; Biomolecules 2018, 8, 78.

31. Chaves, O. A.; Mathew, B.; Cesarin, D. S.; Lakshminarayanan, B.; Joy, M.; Mathew, G. E.; Suresh, J.; Netto-Ferreira, J. C.; J. Mol. Liq. 2017, 242, 1018.

32. Ryan, A. J.; Ghuman, J.; Zunszain, P. A.; Chung, C. W.; Curry, S.; J. Struct. Biol. 2011, 84, 174.

33. Jones, G.; Willet, P.; Glen, R. C.; Leach, A. R.; Taylor, R.; J. Mol. Biol. 1997, 267, 727.

34. Jones, G.; Willett, P.; Glen, R. C.; J. Mol. Biol. 1995, 245, 43.

35. http://www.ccdc.cam.ac.uk/products/life_sciences/gold/, acessada em janeiro de 2019.

36. http://accelrys.com, acessada em janeiro de 2019.

37. Humphrey, W.; Dalke, A.; Schulten, K.; J. Mol. Graphics.1996, 14, 33.

38. Johnson, E.R.; Keinan, S.; Mori, P. S.; Contreras, J. G.; Cohen, A. J.; Yang, W. J. Am. Chem Soc. 2010, 132, 6498.

39. Contreras-Garcia, J.; Johnson, E. R.; Keinan, S.; Chaudret, R; Piquemal, J. P.; Beratan, D. N.; Yang, W.; J. Chem. Theory Comput. 2011, 7, 625.

40. Marques M. A. L.; Botti, S.; Gaz. Fis. 2006, 29, 10.

41. Morgon, N. H.; Custodio, R.; Quim. Nova 1994, 18, 44.

42. http://www.chemkeys.com, acessada em janeiro de 2019.

43. Jensen, F.; Introduction to Computational Chemistry. $3^{\text {rd }}$ ed., Wiley: Chichester, 2017.

44. de Souza, A. R.; Ximenes, V. F.; Morgon, N. H.; Rev. Virtual Quim. 2016, 8,525 . 